\title{
Particle Swarms and Nonextensive Statistics for Nonlinear Optimisation
}

\author{
A.D. Anastasiadis and G.D. Magoulas* \\ School of Computer Science and Information Systems, Birkbeck College, University of London, Malet Street, London \\ WC1E $7 H X, U K$
}

\begin{abstract}
Particle swarm methods are inspired from the dynamics of social interaction and employ information sharing to seek solutions to difficult optimisation problems. In this paper we introduce an approach that combines ideas from particle swarm optimisation (PSO) and the theory of nonextensive statistical mechanics. We develop two algorithms that adopt this approach and conduct an experimental study using benchmark functions to investigate their effectiveness in nonlinear optimisation. Results appear to be promising, as the tested algorithms outperform in most cases the standard PSO and other, recently proposed, PSO variants.
\end{abstract}

Keywords: Particle swarm optimiser, global search methods, statistical mechanics, nonextensive statistical mechanics, nonlinear optimisation.

\section{INTRODUCTION}

In swarm intelligence, the models of social animals are used to create software agents that cooperate to solve complex problems [1]. Simulated annealing [2], genetic and evolutionary algorithms [3], and swarm intelligence [4, 5], have been very popular in nonlinear optimisation and computational intelligence applications. These methods perform global exploration of a search space and have been used extensively where the task can be formulated as a search or optimisation problem.

Among all these methods, the Particle Swarm Optimisation (PSO) algorithm is an evolutionary computation technique for global optimisation [5]. It is inspired by bird flocks' behaviour and was proposed as a population-based method; an efficient alternative to Genetic Algorithms (GA) [6]. Studies and comparisons between PSO and the standard GA $[7,8]$ showed that PSO may exhibit problematic behaviour when it reaches a near optimal solution in several realvalued function optimisation problems. To some extent, PSO could also be considered similar to other evolutionary algorithms $[6,9,10]$. However, it differs from these methods as it does not apply evolutionary operators to the population in order to generate potentially better solutions. Nowadays the selection of PSO in real applications is based on its efficiency in solving a plethora of problems in science and engineering [11-13].

This article introduces new variants of the PSO developed within the framework of statistical mechanics. In particular, the proposed schemes are inspired from the theory of nonextensive statistics [14]. Although, models based on nonextensive statistics have not been used for explaining real bird flocking behaviour, they are often encountered in

*Address correspondence to this author at the School of Computer Science and Information Systems, Birkbeck College, University of London, Malet Street, London WC1E 7HX, UK; E-mail: gmagoulas@dcs.bbk.ac.uk modelling natural systems that are considered to be nonergodic with stationary states, which are metastable and longlived.

In this paper we introduce this approach for perturbing the states of the PSO model. We develop two new PSO variants that use a new type of constriction factor based on nonextensive entropy to control velocities without compromising the diversity of search. We further compare the new variants against the standard PSO, the Fuzzy Particle Swarm Optimiser (FPSO) $[15,16]$, and the Hybrid Particle Swarm Optimiser with mass extinction (HPSO) [17].

The paper is organised as follows. In the next section we describe the fundamentals of the PSO method. Then we proceed with a discussion about basic concepts from statistical mechanics that are important in our approach. The paper continues by introducing central notions from nonextensive statistics, and describing the proposed variants of the PSO method and their nonextensive components. Lastly, an experimental study is presented and the results are discussed. The paper ends with concluding remarks.

\section{BRIEF OVERVIEW OF THE PARTICLE SWARM OPTIMISATION METHOD}

It has been found that PSO is robust in finding good solutions for nonlinear, non differentiable functions with multiple optima and even for time-varying systems [4, 18, 19]. PSO belongs to the class of swarm intelligence algorithms, which are inspired from the social dynamics and emergent behaviour that arises in socially organized colonies. It is a derivative free optimisation method [5], and has been used with reasonable success in many applications especially in cases where traditional optimisation methods "fail"' or when we are looking for a "reasonable" level of "accuracy" within a time limit.

PSO is a population based method, i.e. it exploits a population of individuals to probe for promising regions of the search space, simultaneously. The population is called a 
swarm and the individuals (i.e. the search points) are called particles. The movement of the particles is stochastic; however it is influenced by the particle's own memories as well as the memories of its peers. A minimisation (or maximisation) of the problem topology is found both by a particle remembering its own past best position and the entire swarm's best overall position. A typical PSO algorithm consists of the velocity and position equations, which are given below:

$$
\begin{aligned}
v_{i}(t+1) & =\phi(t) v_{i}(t)+ \\
\eta_{1} r & {\left[p_{i}-x_{i}(t)\right]+\eta_{2} r\left[p_{g(t)}-x_{i}(t)\right] } \\
x_{i}(t+1) & =x_{i}(t)+v_{i}(t),
\end{aligned}
$$

where $i$ is the particle index, $v_{i}(t)$ is the current velocity of the $i$-th particle, $\phi(t)$ is an inertia function (usually a linearly decreasing one), $x_{i}(t)$ is the current position of the $i$-th particle, $p_{i}$ is the position with the best fitness value visited by the $i$-th particle, $p_{g(t)}$ is the particle with the best fitness among all the particles (best position found so far - global version of the PSO), $r$ is a positive constant called acceleration constant, and $n_{1}$ and $n_{2}$ are random numbers uniformly distributed in $[0,1]$.

Many variants of the PSO have been proposed so far following Eberhart and Kennedy's influential work in this area $[5,7]$. A Fuzzy PSO algorithm, proposed by Shi and Eberhart [15], demonstrated reliable performance in many cases. This technique adapts dynamically the inertia weight of the PSO to improve its performance. Another modification of the PSO, the Hybrid Particle Swarm Optimizer (HPSO) [17] produced even better results on unimodal and multimodal functions using mass extinction. Other PSO variants proposed in the literature were equipped with Gaussian mutation [20], or used Gaussian distributions to update the PSO velocities [21]. Lastly, an adaptive Hybrid PSO method [22], which modified the sufficient conditions for the asymptotic stability of the acceleration factor, was proposed to improve the performance of a fuzzy controller for a linear motor driving servo-system.

\section{STATISTICAL MECHANICS}

Statistical mechanics has been the first foundational physical theory in which probabilistic concepts and probabilistic explanation played a fundamental role. Statistical mechanics deals with the macroscopic equilibrium properties of large systems of elements that are subject to the microscopic laws of mechanics [23-25]. It provides a framework for relating the microscopic properties of individual atoms and molecules to the macroscopic properties of materials that can be observed in every day life. It consists of mechanics plus theory of probabilities and has widespread applications, including applications to physical, chemical, biological systems, and other interdisciplinary applications such as optimisation techniques [26-29].

A standard assumption of statistical mechanics is that quantities like energy are "extensive" variables, meaning that the total energy of the system is proportional to the system size; similarly the entropy is also supposed to be extensive. Generally, at least for the energy, this is justified by appealing to the short-range nature of the interactions which hold matter together, form chemical bonds etc. On the other hand, suppose that one deals with long-range interactions, most prominently gravity. Then, one can find that energy is not extensive, which might call for a generalisation of the standard theory.

Statistical mechanics is widely known through Boltzmann's statistical interpolation of the second law of thermodynamics. The celebrated Boltzmann principle is $S=k \ln (W)$, where $k$ is a thermodynamic unit of measurement of entropy and is known as Boltzmann constant $k=1.33 \cdot 10^{-16 \frac{e r g}{C}}$, and $W$, called thermodynamic probability, or statistical weight, or "degree of disorder", which is the total number of microscopic complexions compatible with the macroscopic state of the system.

Boltzmann's Statistical Mechanics is based on the Boltzmann-Gibbs (BG) entropy:

$$
S_{B G}=-k \sum_{i=1}^{W} p_{i} \ln \left(p_{i}\right),
$$

with $\sum_{i=1}^{W} p_{i}=1$ that provides exponential laws for describing stationary states and basic time-dependent phenomena, where $\left\{p_{i}\right\}$ are the probabilities of the microscopic configurations, and $k>0$. This form is known to be the correct entropic form for ergodic systems [30].

Lastly, it is worth to mention an effort to generalise the BG entropy through Renyi entropy [31,32]. This is an extensive entropy for independent systems, which is defined as:

$$
S_{q}^{R} \equiv\left(\ln \sum_{i}^{W} p_{i}^{q}\right) /(1-q),
$$

where $q$ is a continuous parameter.

A general assumption behind statistical mechanics is that the systems being simulated are believed to be ergodic. Ergodicity is a property that is usually postulated for a system in order to make many analytical and computational tasks tractable. The postulate is seldom justified from first principles. Instead, calculations for a system are performed assuming ergodicity. Then if theoretical predictions agree with experiments (actual or numerical), this is taken as evidence that the assumption is valid. Nowadays, many interesting systems are in fact nonergodic. Such complex systems can, in many cases, be handled within the so called Nonextensive Statistical Mechanics [14, 30], a current generalisation of the standard statistical mechanics.

\section{NONEXTENSIVE STATISTICS FOR PARTICLE SWARM OPTIMISATION METHODS}

\section{Nonextensive Statistics}

Boltzmann's Statistical Mechanics is widely used for systems that are in stationary states characterised by thermal equilibrium consistent with ergodicity. Nonextensive Statistical Mechanics is an alternative which has been proposed as 
a way of dealing with anomalous systems through mathematical methods $[14,30]$.

A Nonextensive thermostatistics, which recovers the extensive BG mechanics as a particular case, was proposed in 1988 by Tsallis in order to correctly cover at least some of the known anomalies [14]. Some anomalous systems are considered to be nonergodic systems with stationary states that are metastable and long-lived. Nonextensive Statistical Mechanics exhibits apparent success for certain closed systems as well as in many open systems in biology, economics and other fields.

The Nonextensive Statistical Mechanics is based on the Tsallis entropy, which has been defined [14] as:

$S_{q} \equiv k \frac{1-\sum_{i=1}^{W} p_{i}^{q}}{q-1}, \quad(q \in \mathfrak{R})$

where $W$ is the total number of microscopic configurations, whose probabilities are $\left\{p_{i}\right\}$, and $k$ is a conventional positive constant. When $q=1$, Eq. (4) reproduces the $S_{B G}$ entropic form.

The nonextensive entropy $S_{q}$ achieves its extreme value at the equiprobability $p_{i}=1 / W, \forall i$, and this value is equal to $S_{q}=\frac{W^{1-q}-1}{1-q}[14,30]$. This entropic form possesses the nonextensivity property: for independent systems, it is subextensive for $q>1$, superextensive for $q<1$, and for $q=$ 1 recovers to BG entropy, which is extensive [30].

Recently another generalisation of the BG form, the normalized nonextensive entropy, was independently introduced in [33] and [34]. The form of this entropy is:

$S_{q}^{N} \equiv\left(1-\left[\sum_{i}^{W} p_{i}^{q}\right]^{-1}\right) /(1-q)$.

We should remark at this point that for $q>0$ both the $S_{q}$ and the $S_{B G}$ entropies satisfy the following three important properties, while the Renyi and $S_{q}^{N}$ entropy violate all of them [30]: the first property is concavity, which is related to thermodynamic stability or robustness concerning the fluctuations of energy and other quantities. The second property is stability or continuity that is the experimental robustness, i.e. similar experiments should provide quantitatively similar results. Finally the finiteness of the entropy that characterises the gradual exploration of the available phase space is the third property.

The $S_{q}, S_{q}^{R}, S_{q}^{N}$ entropies, under the same conditions, have in common the optimising distribution as all of them depend on $\sum_{i} p_{i}^{q}$. Hence any of them could be expressed as a function of the other two. All of them lead to the same $q$ exponential optimising distribution [30].

Next we give the relationship between the previously defined entropies and the Tsallis entropy, which will be used in the rest of this article, and in the PSO variants proposed.
Renyi entropy is related through a monotonic function to the nonextensive entropy:

$S_{q}^{R} \equiv\left(\ln \sum_{i}^{W} p_{i}^{q}\right) /(1-q)=\ln \left[1+(1-q) S_{q}\right] /(1-q)$

For $q=1$ the $S_{B G}$ is represented by $S_{1}$, or $S_{1}^{R}$, or finally by $S_{1}^{N}$. Finally the normalised entropy has a strong relationship with the nonextensive entropy by:

$S_{q}^{N} \equiv\left(S_{q}\right) /\left(\sum_{i} p_{i}^{q}\right)=S_{q} /\left[1+(1-q) S_{q}\right]$.

Nowadays the idea of nonextensivity has been used in many applications. Nonextensive statistical mechanics has successfully been applied in physics (astrophysics, astronomy, cosmology, nonlinear dynamics etc.) [35-37], chemistry [38], biology [39], economics [40], computer science [41], and other disciplines [30].

\section{Nonextensive PSO Variants}

The PSO-based methods introduced here are characterised by the nonextensive entropic index $q$. The optimisation of the entropic form in Eq. (4) under appropriate constraints [14], yields for the canonical ensemble:

$p_{i} \propto\left[1-(1-q) \beta E_{i}\right]^{\frac{1}{(1-q)}} \equiv e_{q}^{-\beta E_{i}}$,

where $\beta$ is a Lagrange parameter, $\left\{E_{i}\right\}$ is the energy spectrum, and the q-exponential function is:

$e_{q}^{x} \equiv[1+(1-q) x]^{\frac{1}{(1-q)}}=\frac{1}{[1-(q-1) x]^{\frac{1}{(q-1)}}}$.

The first PSO variant, named the Nonextensive Hybrid PSO (NHPSO), makes use of the $q$-exponential function of Eq. (9) for adaptive searching [41] by incorporating a stochastic constriction factor into the PSO:

$Q_{(T, k)}=e_{q}^{-T(\ln 2) k}=[1-(1-q) T(\ln 2) k]^{\frac{1}{1-q}}$,

where $T$ is the temperature and $k$ indicates iterations (generations). In this version of the NHPSO, particles have no neighbourhood restrictions, the velocity equation uses an inertia weight, as it is in the classical version of the PSO method, and the location of a particle is updated as:

$x_{i d}^{k+1}=x_{i d}^{k}+Q_{i(T, k)} \cdot v_{i d}^{k}$,

where $Q_{i(t, k)}$ is defined by Eq. (10). By tuning the entropic index $q$ and the temperature $T$, the term $Q_{i(t, k)}$ provides an alternative to using a fixed constriction coefficient [42, 43] for balancing two important PSO functions: velocity control and search diversity.

Next, we present another variant that incorporates an adaptive schedule for evolving the influence of the parameters $T$ and $q$. This PSO variant, named the Nonextensive Evolving PSO (NEPSO), is inspired by $[44,45]$ and uses a cooling procedure, which defines a relationship between the 
temperature $T$ and the entropic index $q$ values. The application of cooling helps to regulate better the PSO algorithm. In this way, the behaviour of the NEPSO is getting more stochastic, compared to the PSO and the NHPSO, during the initial stages of the search process and then gradually it becomes more deterministic as the number of iterations increases. Thus, the algorithm will hopefully avoid oscillations and converge faster as the process is getting close to the neighbourhood of a good minimiser.

In NEPSO, the velocity equation and the location of a particle are updated as in NHPSO but the term $Q_{(T, k)}$ is changing dynamically by the cooling schedule that is described in the following equation:

$T=T_{0} \cdot\left[\frac{2^{q-1}-1}{(1+k)^{q-1}-1}\right], q>1$,

where $T_{0}$ is the initial temperature, $T$ is the current temperature, $k$ is the number of iterations (generations), and $q$ is the Tsallis entropic index. This cooling procedure makes the temperature to decrease as a power-law of time, in contrast to the much slower decrease (logarithmic in time) of the $q=$ 1 case.

\section{EXPERIMENTAL STUDY}

We conducted a set of experiments to empirically evaluate the performance of the NHPSO, and NEPSO and compare it with the standard PSO (PSO), the Fuzzy Particle Swarm Optimizer (FPSO) [15, 16], and the Hybrid Particle Swarm Optimizer with mass extinction (HPSO) [17]. All the experiments were performed in Matlab using the revised PSO toolbox Psotb-beta-0.3. The statistical significance of the results has been analysed using the Wilcoxon test [46]; this is a nonparametric method that is considered an alternative to the paired $t$-test. All statements in the tables reported below, refer to a significance level of 0.05 . Statistically significant cases are marked with $(+)$, while a $(-)$ symbol next to a result indicates a case where the difference between the new variants' results and the result of another algorithm is not statistically significant.

We have used three well-studied functions, namely the Rastrigrin, Rosenbrock and Griewank functions. All of them have the same minimum values, which are equal to zero, and they are minimisation problems. The generalized Rastrigrin function is described by the following equation:

$f_{1}(x)=\sum_{i=1}^{n}\left(100\left(x_{i+1}-x_{i}^{2}\right)^{2}+\left(x_{i}-1\right)^{2}\right)$,

whilst the equation of the Rosenbrock function is:

$f_{2}(x)=\sum_{i=1}^{n}\left(x_{i}^{2}-10 \cos \left(2 \pi x_{i}\right)+10\right)$.

Lastly, the generalised Griewank function is defined as:

$$
f_{3}(x)=\frac{1}{4000} \sum_{i=1}^{n} x_{i}^{2}-\prod 10_{i=1}^{n} \cos \left(\frac{x_{i}}{\sqrt{i}}+1\right) \text {. }
$$

\section{Problem Settings}

Following the guideline of $[9,15]$, the asymmetric initialisation method [8] was adopted for the population initialisation. The initialisation ranges and the $V_{\max }$ and $X_{\max }$ values were set as suggested in [15]. Lastly, the fitness function was set as the function value, and the acceleration constant was equal to 2. For the FPSO method we have followed the guideline of [15] and the $V_{\max }$ and $X_{\max }$ values were the same as those in [15].

We have used swarms with $m=\{20,40,80\}$ particles for each function using dimensions $D=\{20,30\}$; the maximum number of generations (iterations) was set to 1500 and 2000 respectively, and 100 different runs were performed for each case.

We did not try to optimise the parameters of the new variants, as the aim of these experiments was to explore the robustness of the approach and the ability to converge despite the perturbations without fine-tuning. Nevertheless we tried different sets of parameters and find out that there is a range of $q$ values $(1.1<q<4.0)$ where the methods perform reasonably well in all cases. As a rule of thumb, when the problem is getting more complicated (high dimensionality), it may be beneficial to apply large values for the $q$, while decrease the value of $q$ as the dimension of the problem reduces. Below we give some typical examples of behaviour from the combinations tried.

Table 1. Average Fitness Values for the Rastrigrin Function

\begin{tabular}{|c|c|c|}
\hline Algorithms & $\begin{array}{l}m=20 \\
D=20\end{array}$ & $\begin{array}{c}m=20 \\
D=30\end{array}$ \\
\hline PSO & $25.5(+)$ & $51.2(+)$ \\
\hline FPSO & $25.2(+)$ & $58.4(+)$ \\
\hline HPSO & $24.9(-)$ & $45.5(-)$ \\
\hline NHPSO & 25.0 & 44.2 \\
\hline \multirow[t]{2}{*}{ NEPSO } & 24.1 & 44.1 \\
\hline & $\begin{array}{l}m=40 \\
D=20\end{array}$ & $\begin{array}{l}m=40 \\
D=30\end{array}$ \\
\hline PSO & $18.5(+)$ & $41.2(-)$ \\
\hline FPSO & $18.0(+)$ & $40.2(-)$ \\
\hline HPSO & $16.0(+)$ & $31.1(-)$ \\
\hline NHPSO & 15.2 & 40.6 \\
\hline \multirow[t]{2}{*}{ NEPSO } & 14.2 & 38.6 \\
\hline & $\begin{array}{l}m=80 \\
D=20\end{array}$ & $\begin{array}{l}m=80 \\
D=30\end{array}$ \\
\hline PSO & $13.6(+)$ & $33.3(+)$ \\
\hline FPSO & $12.8(+)$ & $29.5(+)$ \\
\hline HPSO & $10.1(+)$ & $24.0(+)$ \\
\hline NHPSO & 9.0 & 22.5 \\
\hline NEPSO & 12.1 & 23.6 \\
\hline
\end{tabular}




\section{Presentation of Results}

Table 1 shows results for the Rastrigrin function. The temperature was set at $T=0.0001$ and $q=2$ for the NHPSO; $T=0.001$ and $q=2.5$ for the NEPSO. The new methods outperform the standard PSO in most cases, and present slight improvement compared to the other variants.

Table 2. Average Fitness Values for the Rosenbrock Function

\begin{tabular}{|c|c|c|}
\hline Algorithms & $\begin{array}{l}m=20 \\
D=20\end{array}$ & $\begin{array}{l}m=20 \\
D=30\end{array}$ \\
\hline PSO & $252.6(+)$ & $322.4(+)$ \\
\hline FPSO & $208.3(+)$ & $203.8(+)$ \\
\hline HPSO & $192.5(+)$ & $188.0(+)$ \\
\hline NHPSO & 141.5 & 180.9 \\
\hline \multirow[t]{2}{*}{ NEPSO } & 120.5 & 160.0 \\
\hline & $\begin{array}{l}m=40 \\
D=20\end{array}$ & $\begin{array}{l}m=40 \\
D=30\end{array}$ \\
\hline PSO & $180.97(+)$ & $269.7(+)$ \\
\hline FPSO & $163.88(+)$ & $175.0(+)$ \\
\hline HPSO & $158.97(+)$ & $122.9(+)$ \\
\hline NHPSO & 64.00 & 111.0 \\
\hline \multirow[t]{2}{*}{ NEPSO } & 74.50 & 116.4 \\
\hline & $\begin{array}{l}m=80 \\
D=20\end{array}$ & $\begin{array}{l}m=80 \\
D=30\end{array}$ \\
\hline PSO & $97.2(+)$ & $205.5(+)$ \\
\hline FPSO & $56.0(+)$ & $124.4(+)$ \\
\hline HPSO & $48.9(+)$ & $164.4(+)$ \\
\hline NHPSO & 34.7 & 70.1 \\
\hline NEPSO & 44.5 & 60.1 \\
\hline
\end{tabular}

Table 2 shows the average fitness for tests in the Rosenbrock function; $q=1.5$ and $T=0.001$ for the NHPSO and $T=0.01$ for the NEPSO.

Figs. (1) and (2) show that the reduction of the average fitness per generation by the new variants is better than the standard PSO; these curves were produced by taking the average of 20 runs using a population of 20 particles and a problem dimensionality of 20 .

The last test, Table $\mathbf{3}$, highlights the performance of the new variants in the Griewank function; $q=1.5$ and $T=0.01$ for the NEPSO; $T=0.0001$ and $q=1.5$ for the NHPSO.

Fig. (3) exhibits the reduction of the average fitness calculated over 20 runs; these results were produced by a population of 20 particles and a dimensionality of 20 . It appears the new variants offer a viable alternative even without fine tuning their parameters.

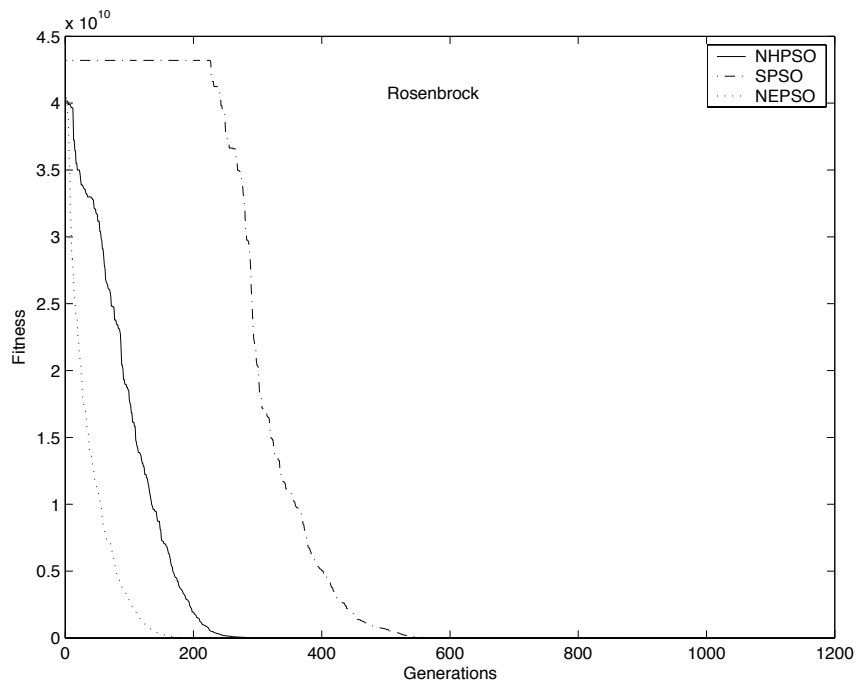

Fig. (1). The average fitness performance for Standard PSO (SPSO) and the new variants in the Rosenbrock functions.

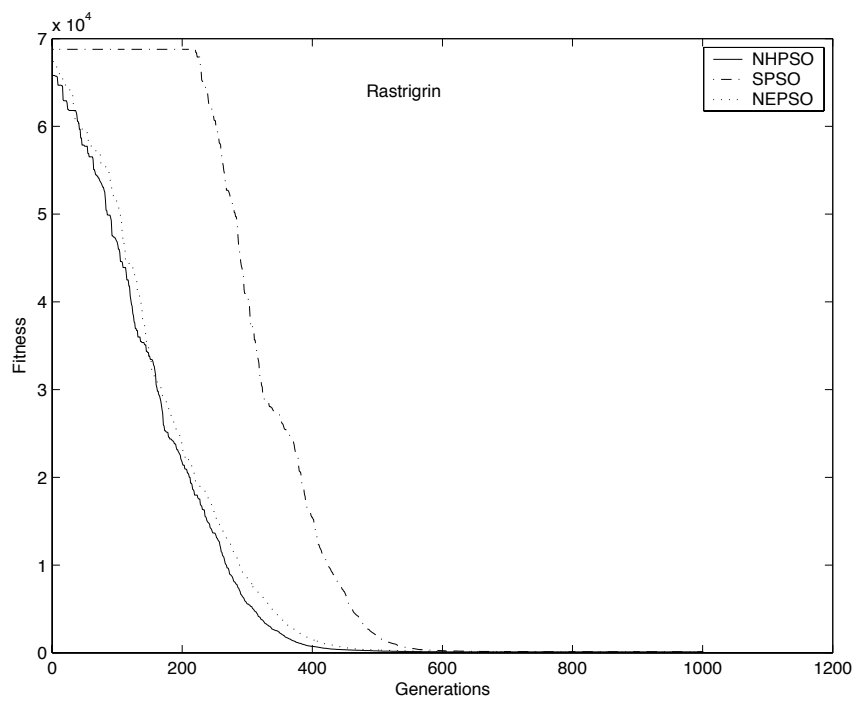

Fig. (2). The average fitness performance for Standard PSO (SPSO) and the new variants in the Rastrigrin function.

\section{CONCLUDING REMARKS}

In this paper, we proposed an approach that equips Particle Swarm Optimisation algorithms with mechanisms inspired by principles of nonextensive statistics. The two variants introduced in the paper exploit the nonextensive entropic index $q$ and adaptive temperature control to regulate the stochasticity of the methods.

The nonextensive components introduced in the paper could also be used to equip other PSO variants, such as the HPSO method that performed well in many cases in our experiments. This kind of investigation as well further testing in different classes of problems is at the core of our research agenda in the near future in order to fully explore the advantages of these techniques, optimise the parameter values and identify possible limitations. 
Table 3. Average Fitness Values for the Griewank Function

\begin{tabular}{|c|c|c|}
\hline Algorithms & $\begin{array}{l}m=20 \\
D=20\end{array}$ & $\begin{array}{l}m=20 \\
D=30\end{array}$ \\
\hline PSO & $0.027(+)$ & $0.0150(+)$ \\
\hline FPSO & $0.026(+)$ & $0.0140(+)$ \\
\hline HPSO & $0.025(+)$ & $0.0125(-)$ \\
\hline NHPSO & 0.024 & 0.0120 \\
\hline \multirow[t]{2}{*}{ NEPSO } & 0.022 & 0.0120 \\
\hline & $\begin{array}{l}m=40 \\
D=20\end{array}$ & $\begin{array}{l}m=40 \\
D=30\end{array}$ \\
\hline PSO & $0.032(+)$ & $0.014(+)$ \\
\hline FPSO & $0.029(+)$ & $0.012(+)$ \\
\hline HPSO & $0.027(+)$ & $0.011(-)$ \\
\hline NHPSO & 0.021 & 0.009 \\
\hline \multirow[t]{2}{*}{ NEPSO } & 0.028 & 0.010 \\
\hline & $\begin{array}{l}m=80 \\
D=20\end{array}$ & $\begin{array}{l}m=80 \\
D=30\end{array}$ \\
\hline PSO & $0.029(+)$ & $0.017(+)$ \\
\hline FPSO & $0.028(+)$ & $0.014(+)$ \\
\hline HPSO & $0.027(-)$ & $0.013(+)$ \\
\hline NHPSO & 0.027 & 0.011 \\
\hline NEPSO & 0.027 & 0.010 \\
\hline
\end{tabular}

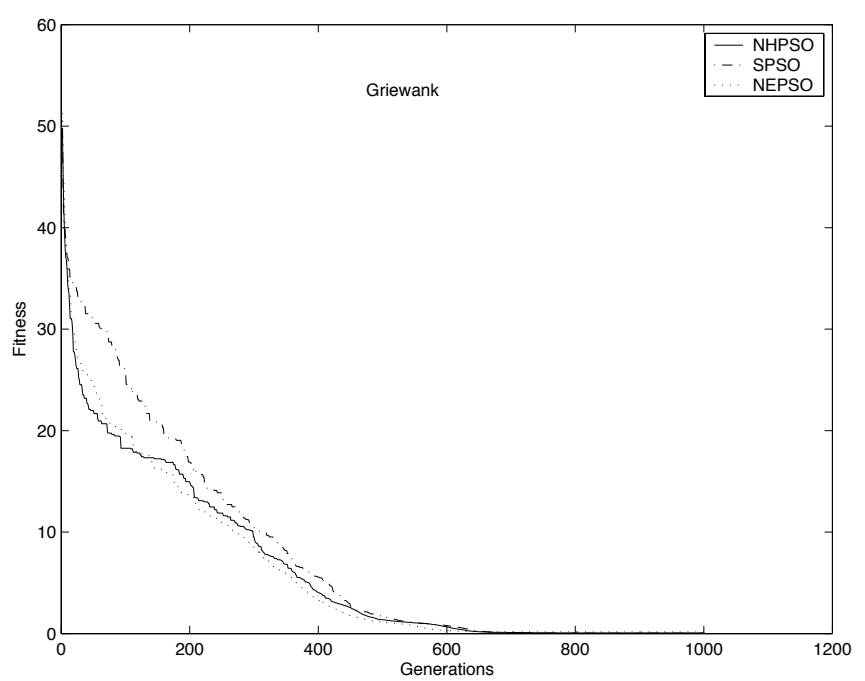

Fig. (3). The average fitness performance for Standard PSO (SPSO) and the new variants in the Griewank function.

\section{REFERENCES}

[1] B. Bonabeau, M. Dorigo, and G. Thraulaz, Swarm intelligence: from natural to artificial systems, Oxford University Press, Oxford, 1999.

[2] S. Kirkpatrick, C. D. Gelatt Jr., and M. P. Vecchi, "Optimization by simulated annealing", Science, vol. 220, pp. 671-680, 1983.

[3] Z. Michalewicz, Genetic Algorithms + Data Structures = Evolution Programs, Springer, New York, 1996.
[4] R. Eberhart, P. K. Simpson, and R. W. Dobbins, Computational Intelligence PC Tools, Academic Press Professional, Boston, MA, 1996.

[5] J. Kennedy, and R. Eberhart, "Particle Swarm Optimization" Proceedings of the IEEE International Conference on Neural Networks, 1995, pp. 1942-1948.

[6] A. H. Wright, "Genetic Algorithms for Real Parameter Optimization", In: Foundations of Genetic Algorithms, G.J.E Rawlins (Ed.), Morgan Kaufmann, 1991.

[7] R. Eberhart, and Y. Shi, "Comparison between genetic algorithms and particle swarm optimization", Proceedings of the 7th Annual Conference on Evolutionary Programming, 1998, pp. 611-616.

[8] P. J. Angeline, "Evolutionary Optimization versus Particle Swarm Optimization: Philosophy and Performance Differences", In: Evolutionary Programming VII, V. William Porto, N. Saravanan, Donald E. Waagen, A. E. Eiben (Ed.), Lecture Notes in Computer Science vol. 1447, Springer, 1998, pp. 601-610.

[9] D. B. Fogel, Evolutionary Computation: Toward a New Philosophy of Machine Intelligence, IEEE Press, New York, 1995.

[10] J. Kennedy, and R. Eberhart, Swarm Intelligence, Morgan Kaufmann Publishers, 2001.

[11] A. E. Ahmed, L. T. Germano, and Z. C. Antonio, "A hybrid particle swarm optimization applied to loss power minimization", IEEE Trans. Power Sys., vol. 20, pp. 859-866, 2005.

[12] F.-Q. Zhao, Q.-Y. Zhang, and Y.-H. Yang, "A Hybrid SelfAdaptive PSO Algorithm and its Applications for Partner Selection in Holonic Manufacturing System", Proceedings of the International Conference Machine Learning and Cybernetics, 2006, pp. 2536-2541.

[13] H.-M. Feng, C.-Y. Chen, and F. Ye "Adaptive hyper-fuzzy partition particle swarm optimization clustering algorithm", Cybernet. Syst., vol. 37, pp. 463-479, 2006.

[14] C. Tsallis, "Possible Generalization of Boltzmann-Gibbs Statistics", J. Statist. Phys., vol. 52, no. 1-2, pp. 479-487, 1988.

[15] Y. Shi, and R. Eberhart, "Fuzzy adaptive particle swarm optimization", Proceedings IEEE International Conference on Evolutionary Computation, 2001, pp. 101-106.

[16] Y. Shi, and R. Eberhart, "Particle swarm optimization with fuzzy adaptive inertia weight", Proceedings of the Workshop on Particle Swarm Optimization, 2001.

[17] X.-F. Xie, W.-J. Zhang, and Z.-L. Yang, "Hybrid Particle Swarm Optimizer with Mass Extinction", Proceedings of the IEEE International Conference on Communication, Circuits and Systems, 2002, vol. 2, pp. 1170-1174.

[18] R. Eberhart, and Y. Shi, "Evolving Artificial Neural Networks", Proceedings of the International Conference on Neural Networks and Brain, 1998, PL5-PL13.

[19] Y. Fukuyama and H. Yoshida, "A Particle Swarm Optimization for Reactive Power and Voltage Control in Electric Power Systems", Proceedings of the IEEE Congress on Evolutionary Computation, 2001, vol. 1, pp. 87-9.

[20] N. Higashi, and H. Iba, "Particle swarm optimization with Gaussian mutation", Proceedings of the IEEE Swarm Intelligence Symposium, Indianapolis, IN, 2003, pp. 72-79.

[21] R. A. Krohling, F. Hoffmann, and L. dos Santos Coelho, "Coevolutionary particle swarm optimization for min-max problems using gaussian distribution", Proceedings of the IEEE Congress on Evolutionary Computation, 2004, vol. 1, pp. 959-964.

[22] J. Chen, F. Pan, and T. Cai, "Acceleration factor harmonious particle swarm optimizer”, Int. J. Autom. Comput., vol. 3, pp. 41-46, 2006.

[23] E. Engel, and C. Van den Broeck, Statistical Mechanics of Learning, Cambridge University Press, 2001.

[24] D. A. Lavis, "Is equilibrium a useful concept in statistical mechanics?", Proceedings of the Conference on Philosophical and Foundational Issues in Statistical Physics, Utrecht, November 2003.

[25] J. L. Lebowitz, "Statistical mechanics: A selective review of two central issues", Rev. Mod. Phys., vol. 71, no. 2, pp. 346-357, 1999.

[26] R. Fondecave, and F. Brochard-Wyart, "Application of statistical mechanics to the wetting of complex liquids", Phys. A: Statist. Mechan. Appl., vol. 274, no. 1-2, pp. 19-29, 1999.

[27] R. N. Mantegna, Z. Palágyi, and H. E. Stanley, "Applications of statistical mechanics to finance", Phys. A: Statist. Mechan. Appl., vol. 274, no. 1-2, pp. 216-221, 1999. 
[28] T. Vicsek, A. Czirók, I. J. Farkas, and D. Helbing, "Application of statistical mechanics to collective motion in biology", Phys. A: Statist. Mechan. Appl., vol. 274, no. 1-2, pp. 182-189, 1999.

[29] M. Wolf, "Applications of statistical mechanics in number theory", Phys. A: Statist. Mechan. Appl., vol. 274, no. 1-2, pp. 149-157, 1999.

[30] M. Gell-Mann, and C. Tsallis (eds.), Nonextensive EntropyInterdisciplinary Applications, Oxford University Press, New York, 2004.

[31] A. Renyi, Wahrscheinlichkeitsrechmung, Deutscher Verlag der Wissenschften, Berlin, 1966.

[32] A. Wehrl, "General properties of entropy", Rev. Mod. Phys., vol. 50, no. 2, pp. 221-260, 1978.

[33] P. T. Landsberg, and V. Vedral, "Distributions and channel capacities in generalized statistical mechanics", Phys. Lett. A, vol. 247, pp. 211-217, 1998.

[34] A. K. Rajagopal, and S. Abe, "Implications of Form Invariance to the Structure of Nonextensive Entropies", Phys. Rev. Lett., vol. 83, pp. 1711-1714, 1999.

[35] H. Shibata, "Statistics of phase turbulence II", Phys. A: Statist. Mechan. Appl., vol. 317, no. 3-4, pp. 391-400, 2003.

[36] W. H Siekman., "The entropic index of the planets of the solarsystem”, Chaos, Soliton Fract., vol. 16, no. 1, pp. 119-124, 2003.

[37] A. Taruya, and M.-A. Sakagami, "Gravothermal catastrophe and Tsallis generalized entropy of self-gravitating systems", Phys. A: Statist. Mechan. Appl., vol. 307, no. 1-2, pp. 185-206, 2002.
[38] P. Serra, A. F. Stanton, S. Kais, and R. E. Bleil, "Comparison study of pivot methods for global optimization", J. Chem. Phys., vol. 106, no. 17, pp. 7170-7177, 1997.

[39] A. Upadhyaya, J. Rieu, J. A. Glazier, and Y. Sawada, "Anomalous diffusion and non-Gaussian velocity distribution of Hydra cells in cellular aggregates", Phys. A: Statist. Mechan. Appl., vol. 293, no. 3-4, pp. 549-558, 2001.

[40] C. Tsallis, C. Anteneodo, L. Borland, and R. Osorio, "Nonextensive statistical mechanics and economics", Phys. A: Statist. Mechan. Appl., vol. 324, no. 1-2, pp. 89-100, 2003.

[41] G. D. Magoulas, and A. D. Anastasiadis, "Approaches to Adaptive Stochastic Search Based on the Nonextensive q-Distribution", Int. J. Bifurcat. Chaos, vol. 16, pp. 2081-2091, 2006.

[42] M. Clerc, "The swarm and the queen: towards a deterministic and adaptive particle swarm optimization", Proceedings of the Congress of Evolutionary Computation, IEEE Press, New York, 1999, pp. 1951-1957.

[43] R. Eberhart, and Y. Shi, "Comparing inertia weights and constriction factors in particle swarm optimization", Proceedings of the Congress on Evolutionary Computation, San Diego, CA, 2000, pp. 84-88.

[44] A. D. Anastasiadis, and G. D. Magoulas, "Evolving stochastic learning algorithms based on Tsallis entropic index", Eur. Phys. $J$. $B$, vol. 50, pp. 277-283, 2006.

[45] C. Tsallis, and D.A. Stariolo, "Generalized Simulated Annealing", Phys. A: Statist. Mechan. Appl., vol. 233, pp. 395-406, 1996.

[46] G. Snedecor, and W. Cochran, Statistical Methods, Iowa State University Press, 8th edition, 1989.

(C) Anastasiadis and Magoulas; Licensee Bentham Open.

This is an open access article distributed under the terms of the Creative Commons Attribution License (http://creativecommons.org/licenses/by/2.5/), which permits unrestrictive use, distribution, and reproduction in any medium, provided the original work is properly cited. 